\section{Cahiers de Narratologie}

Analyse et théorie narratives

8 | 1997

Création de l'espace et narration littéraire

\title{
Lieux éloquents, lieux muets dans Béatrix de Balzac
}

Aline Mura

\section{OpenEdition}

Journals

Édition électronique

URL : http://journals.openedition.org/narratologie/11617

DOI : 10.4000/narratologie. 11617

ISSN : 1765-307X

Éditeur

LIRCES

Édition imprimée

Date de publication : 1 décembre 1997

Pagination : 285-294

ISBN : 291089746X

ISSN : 0993-8516

\section{Référence électronique}

Aline Mura, «Lieux éloquents, lieux muets dans Béatrix de Balzac », Cahiers de Narratologie [En ligne], 8 | 1997, mis en ligne le 15 décembre 2020, consulté le 25 février 2021. URL : http:// journals.openedition.org/narratologie/11617; DOI : https://doi.org/10.4000/narratologie.11617

Ce document a été généré automatiquement le 25 février 2021.

Article L.111-1 du Code de la propriété intellectuelle. 


\title{
Lieux éloquents, lieux muets dans Béatrix de Balzac
}

\author{
Aline Mura
}

1 Il y a chez Balzac, on le sait, des "personnages reparaissant ». Mais il y a aussi, et on le sait moins, des lieux reparaissant. Ils peuvent être éloquents ou muets, pour le narrateur, le personnage et le lecteur.

2 Nous tenterons de déterminer quels sont leurs significations, leurs enjeux et leurs effets du point de vue du lecteur, et ce, à un triple niveau : extratextuel, intertextuel et intra-textuel. Nous proposons de repérer dans le roman de Béatrix des occurrences de ces trois types de fonctionnement: l'hôtel du Guénic à Guérande sert de point de convergence à un savoir extratextuel (essentiellement historique) ; le faubourg SaintGermain à Paris se pose comme référent d'une compétence idéologique (à la fois extratextuelle et intertextuelle); le château des Touches, situé dans le village breton, peut être perçu comme un espace ambivalent qui recueille à la fois des signes extratextuels et textuels ; enfin, ces mêmes lieux revus par Calyste du Guénic et Sabine de Grandlieu, sont dotés d'une signification accrue - construite au fil de la narration et particulièrement éloquente pour le lecteur. Il arrive que ces trois niveaux interfèrent.

Ajoutons que les lieux - réels mais fictionnalisés - deviennent proprement « un espace romanesque " dans ce dernier cas. Ils permettent, rétrospectivement d' «identifier des continuités sémantiques qui font du texte lu un ensemble cohérent $»^{1}$, autrement dit des « isotopies ». Nous réservons donc le terme de "lieux éloquents » pour les données spatiales qui organisent le sens et la signification du texte (c'est-à-dire le déchiffrement opéré par le lecteur et l'effet produit sur son existence même, selon la distinction de Paul Ricœur). Cela dit, les lieux entièrement muets n'existent pas : ils parlent toujours à quelque instance de la narration, mais nous privilégions ici ceux qui sont coréférentiels, qui parlent au lecteur et s'élaborent - en se singularisant - au sein de la narration. Ils se taisent partiellement lorsque leur sens est déjà pertinent, dans un espace extra-diégétique.

Or, Béatrix est souvent considérée par les critiques comme un roman mal construit, issu $d^{\prime}$ « un replâtrage $»^{2}$. En effet, composé en deux temps -1839 et 1845 , autour de 
personnages, de lieux et d'intrigues essentiellement différents, il porte préjudice, semble-t-il, à l'exigence balzacienne d' « unité de composition ».

Les déplacements des personnages et les points d'ancrage du texte constituent peutêtre le seul lien possible entre les éléments de la mosaïque dans ce récit composite : la construction du sens passerait par l'observation d'un espace loquace. C'est en tous cas notre hypothèse.

\section{Une compétence idéologique :}

6 Elle renvoie à des structures axiologiques de l'œuvre et facilite la mise en place de l'édifice romanesque.

7 Des scénarios attendus se réalisent. La présentation archéologique et les descriptions auxquelles elle donne lieu (alentours, village, hôtel, habitants) disposent les signes nécessaires à l'intelligence de l'histoire. La formule de P. Hamon s'applique particulièrement bien au baron du Guénic, vieux général au repos qui fait corps avec ces monuments, véritables vestiges du passé : " "l'effet-personnage" d'un récit n'est peutêtre que la somme, la résultante d'un certain nombre d" "effets-descriptifs" disséminés dans l'énoncé »".

8 Ce roman balzacien - fait exceptionnel - ne repose pas sur une construction analeptique. En effet, la ville de Guérande et l'hôtel du Guénic parlent assez haut pour que le récit puisse se taire sur ses antécédents. Le discours archéologique tient lieu de narration. Les monuments - au sens étymologique - sont en eux-mêmes figures du passé : ils suscitent une lecture rétrospective que le narrateur confirme explicitement :

Encore quelques années, ces cités originales seront transformées et ne se verront plus que dans cette iconographie littéraire ${ }^{5}$.

Ils relatent la féodalité, le conservatisme et le respect des traditions. Fidèle à la logique de cet espace, le baron du Guénic est un des acteurs principaux de la guerre de Vendée. Le récit des événements peut être elliptique puisque les lieux sont éloquents, sur le plan idéologique - et donc narratif. En somme, cette longue présentation, caution obligée d'un texte qui se pose comme "réaliste", revêt une valeur sémiotique indéniable. Mais elle fait appel à un savoir normalement préexistant. Le narrateurauteur se plaint d'ailleurs de l'ignorance du lecteur et des lacunes de l'Histoire officielle, qui néglige l'histoire des mœurs. Le roman se pose alors comme substitut d'un savoir extralittéraire déficient. L'éloquence du lieu reste donc d'abord extradiégétique.

10 L'hôtel du Guénic, devient en revanche, un lieu modalisé lorsque Calyste apparaît. Par son comportement en effet, ce "magnifique rejeton de la plus vieille race bretonne ${ }^{6}$ va faire mentir les prédictions inscrites dans ces murs. Il perturbera les scénarios préparés et les structures axiologiques afférentes. Calyste vit dans ces lieux ; pourtant il ne cesse de partir, sa mère guettant son retour. Il refuse également d'épouser les valeurs inscrites dans cette vénérable maison et le destin tout tracé qu'elle lui indique. Cet univers référentiel, particulièrement explicite, s'opacifie alors : le lecteur est dérouté par l'attitude négative du jeune homme, - ce décalage étant bien sûr un levier narratif efficace, qui ménage une attente déceptive et déjoue la compétence idéologique du récepteur. Le personnage, en rupture avec cette société et cet espace prédéterminé, est contraint de trouver un espace ailleurs: aristocrate marginalisé par les nouvelles 
données de la politique, le jeune baron oscille d'un lieu à l'autre, en porte-à-faux et excentré. A Paris, il trouve pourtant ses pairs; mais là encore l'inadéquation réapparaît.

11 Le faubourg Saint Germain est l'emblème de la haute société parisienne. Ce lieu est si fortement épinglé comme tel dans l'idéologie de l'époque que les descriptions ont quasiment disparu. La référence extratextuelle joue ici pleinement et le récit reste allusif. Le lecteur, passant d'un lieu à l'autre par l'entremise de Calyste, voit l'opposition Paris/province réactivée. L'espace peut être dit muet dans la mesure où il ne donne pas lieu à une présentation circonstanciée et où il n'est pas chargé sémiotiquement, de façon spécifique, dans ce roman : il s'agit tout simplement d' "un des plus beaux hôtels de la rue de Bourbon $»^{7}$, acheté par Mlle des Touches à Calyste pour qu'il s'y installe avec sa nouvelle épouse, Sabine de Grandlieu. La jeune femme est le type représentatif de ce milieu et de ce lieu : elle est, quant à elle, dotée d'un superlatif évasif (c'est " une des plus belles et des plus charmantes filles de la société parisienne $\left.{ }^{8}\right)$. Il est rare de voir Balzac aussi économe dans ses descriptions et ses portraits. C'est là une preuve de la pertinence des noms - ancrés dans un contexte sociologique précis avant même que ne s'écrive le récit.

12 A ce mutisme du lieu correspond à l'évidence une compétence extratextuelle suffisante. Le « décor " renvoie de surcroît, dans ce cas, à une connaissance intertextuelle, celle que possède le lecteur de La comédie humaine.

\section{Une compétence intertextuelle}

Le faubourg Saint-Germain est en effet un lieu reparaissant. Force est de reconnaître qu'il représente à la fois une référence historique répertoriée et un stéréotype du système balzacien. Citons, à titre indicatif, La duchesse de Langeais (1834), Le père Goriot (1835), et « Un grand homme de province à Paris » in Illusions perdues (1839). Ce quartier de Paris est presque devenu un lieu commun dans la nomenclature de Balzac.

14 Quant à la côte bretonne et au baron du Guénic, ils renvoient au texte le plus ancien qui figure dans La comédie humaine, à savoir Les chouans (1829). L'hôtel du Guénic est toutefois un lieu non reparaissant et il fait logiquement l'objet d'une description minutieuse dans le roman qui nous occupe. Une poétique cohérente de l'espace se met donc progressivement en place : le récit répare exactement les lacunes de l'Histoire et les ellipses des autres textes; il s'ajuste parfaitement aux compétences ou ignorances supposées du destinataire. Éloquent, lorsqu'il tient lieu de savoir; discret, quand il serait superfétatoire, le lieu balzacien est en intime corrélation avec les modalités d'un récit efficace sur le plan de la lisibilité et de l'interprétation. Cependant, il ne devient un élément sémiotique prépondérant que lorsque le texte le redéfinit ou l'invente. Le personnage est alors un agent indispensable dans la construction du sens : le lecteur le suit aveuglément.

\section{Une compétence textuelle}

Or le rôle du récepteur s'avère à ce moment-là beaucoup plus actif : celui-ci participe indéniablement à l'élaboration du sens et de la cohérence textuelles. Il s'agit d'une "opération [...] ontologique " : le lecteur fait en somme l'expérience de soi à travers 
celle de l'autre. Des scénarios non attendus apparaissent alors, à travers des lieux fortement modalisés.

Le château des Touches, espace hybride, à la fois extratextuel et intra-textuel, est exemplaire à cet égard. En effet, des éléments extérieurs faisant appel à un savoir antérieur (la Bretagne, Paris), suivant un parcours axiologique prédéterminé, constituent ce lieu. Mais la dualité, perceptible dans le même espace, représente une audace qui transcende les données référentielles. Le lieu parle un autre langage, celui que ce texte-là lui donne. Mlle des Touches, la propriétaire a conservé le caractère typiquement breton de l'édifice dans l'architecture (elle reste attachée aux valeurs de son pays natal); mais elle a rassemblé dans ses appartements privés les signes du raffinement parisien : «toutes les choses élégantes, riches, somptueuses, délicates »"

Calyste, ébloui par ce monde fascinant de la culture et du luxe, est là encore en décalage : on le trouve souvent dans les escaliers, sur le seuil ou dans les antichambres. Il est condamné à errer, indécis, d'un espace à l'autre jusqu'à ce qu'une révélation s'impose lors de son retour à Guérande. Dans la bibliothèque de Mlle des Touches, alias Camille Maupin, il surprend les propos confidentiels tenus par Camille et Claude Vignon: " "Je ne dois pas vous laisser ignorer que je suis là", dit-il »"1. Il restera aussi à la porte de la maison de Béatrix, à Paris. Il n'appartient à aucun lieu; il est «neutre », ni l'un ni l'autre, susceptible d'incarner une position ne puis une autre sans jamais se laisser appréhender par des désignations univoques. Calyste représente peut-être la « voix » narrative par laquelle passe la position du lecteur (il est à la lisière de tous les lieux, insolite, déplacé). Il en perçoit les effets sans jamais être un acteur principal, sur le devant de la scène. Il passe de l'un à l'autre et finalement donne à lire un sens «obvie »" ${ }^{12}$ cohérent, émanant de l'opération de lecture elle-même.

Or le retour à l'hôtel du Guénic et aux Touches, effectué par Calyste et Sabine, déclenche une révélation tardive mais lumineuse du sens de l'espace. Le lecteur procède alors à une analyse herméneutique des différents acquis narratifs. L'espace se construit là sous ses yeux, plus éloquent que jamais, et délivre son secret - différé et dissimulé - dans les replis du texte. Modalisés et intériorisés, les lieux revisités jouent pleinement leur rôle sémiotique. L'hôtel du Guénic, les rochers de Guérande et la propriété des Touches sont marqués du sceau de l'enfance, du déjà là perdu et retrouvé ; mais ils recèlent aussi de nouveaux messages. Le couple joue respectivement "le rôle d'une châtelaine adorée de ses vassaux $~^{13}$ et d'un " joli seigneur $»^{14}$ : ils traversent ces paysages et cette demeure, sans les investir dans la mesure où ils correspondent parfaitement à l'attente sociologique et narrative.

19 Mais, lorsqu'ils arrivent aux Touches, ils sont plongés dans un passé plus récent, qui éveille des échos personnels. Espaces du souvenir et des désirs refoulés, le domaine fonctionne comme la chambre noire du récit. Le cabinet de Barbe-Bleue punit de mort les indiscrets; il ravive l'image de Béatrix, rivale potentielle de Sabine: "L'a-t-il oubliée ? $»^{15}$ Faisant écho à "L'as-tu bien oubliée ? ${ }^{16}$ Le voyage de «la lune de miel » (premier titre de cette partie du roman ${ }^{17}$ ) est donc terni par la force du passé - présent dans les lieux. Sabine obtient de Calyste, dans un premier temps, le récit de sa passion pour Béatrix de Rochefide et elle décide de revoir les Touches afin de mettre à l'épreuve les sentiments actuels de son mari. Or, Calyste retrouve dans ce "lieu fatal $»^{18}$ des souvenirs intacts. Le récit ne cesse de disposer des signes proleptiques, comme autant de mises en garde : par la bouche de Camille, désormais cloîtrée dans le couvent des Dames de la Visitation, la jeune femme a entendu ce conseil : 
«Enfant! Les Touches sont pour toi le cabinet de Barbe-Bleue, car il n'y a rien de plus dangereux que de réveiller une passion qui dort $»^{19}$. projet.

Nous autres femmes, qui sommes encore un peu jeunes filles, il suffit qu'on nous dise : "Voici une clef rouillée de souvenirs parmi toutes celles de votre palais, entrez partout, jouissez de tout, mais gardez-vous d'aller aux Touches! » pour que nous entrions là, les pieds chauds, les yeux allumés de la curiosité d'Eve ${ }^{20}$.

La métaphore filée élucide la fonction de ce huis clos, véritable sanctuaire du péché. La logique herméneutique de la retenue et du dévoilement organise le parcours lectoral : voir, c'est savoir - dire devenant presque superflu. Le cabinet de Barbe-Bleue est doté d'une valeur performative dans la mesure où il suffit d'y pénétrer pour comprendre et souffrir. Le faire dispense le sens. Ce lieu ne cristallise pas tout le refoulé de La comédie humaine mais il rétablit l'équilibre de ce roman «replâtré». Perçu comme éclaté, le texte balzacien peut donc abriter une zone d'ombre, susceptible de recueillir les doutes et les fautes dissimulés jusque là. Pôle de silence qui rassemble les débordements disséminés ailleurs, il représente un modèle privilégié du fonctionnement de l'écriture balzacienne. Tout roman n'a-t-il pas son cabinet de Barbe-Bleue - qu'il suppose sans le nommer?

De fait, l'espace signale, seulement mais fermement, l'émergence d'un ordre de lecture : le récit - réversible - se lirait à rebours, de Paris aux Touches (c'était d'ailleurs la présentation de la première version (1838)). C'est aussi le trajet effectué avant Calyste et Sabine - par Camille! Mais il s'agit là d'une péripétie entièrement extra-diégétique, réduite à un compte-rendu rétrospectif et lacunaire.

Ainsi les lieux qui avaient enseveli la passion délétère la mettent-ils au grand jour. Ils tiennent le langage du désir, qui est aussi celui de la répétition : leur éloquence devient imprudence. Béatrix, coupable d'adultère, y avait trouvé un refuge ; Camille, femmeauteur trop peu femme, s'était mise à l'abri, au creux des rochers; quant à Calyste, il dévoile cette intimité, à son insu. Ballotté jusque là entre des mondes et des pulsions contraires, errant éternellement - à la recherche de la Béatrice dantesque, évanescente et parodiée dans le roman - il trouve enfin le bout de son chemin. La performance textuelle repose donc sur la mémoire: «tout artifice mnémotechnique est indiscutablement un phénomène de pertinence sémiotique ${ }^{21}$, affirme Umberto Eco. Le château des Touches, lieu de réminiscences, fait jouer le processus de la mémoire affective et involontaire. Il devient au contraire pour le lecteur le point d'ancrage d'une anamnèse puisqu'il lit dans l'espace l'avenir des personnages, la disposition des signes narratifs et les effets d'un tel récit, transposé dans le réel. Il s'agit donc bien d'un «sens » et d'une "signification» - pour reprendre la distinction de Paul Ricœur - qui émanent de ce parcours. Les lieux ont sollicité cette fois une compétence strictement textuelle tant leur performance est inattendue et leur force perlocutoire décisive.

Cela dit, le texte ne s'en tient pas là. Les masques sont tombés et l'espace occulté a été violé. Or, la logique du secret consiste à différer sans cesse la révélation. Le récit se perd si le lecteur trouve d'emblée "le sens caché $»^{22}$. Les lieux ont été si explicites qu'ils ont brisé le tabou. Mais le roman ménage d'autres surprises et l'espace d'autres enseignements : certes, le couple Calyste/Sabine surmontera cette épreuve. Le Cabinet de Barbe-Bleue sera ouvert; il produira l'effet escompté mais la constellation familiale organisera un complot pour provoquer la rupture de Béatrix et de Calyste, le retour de 
Béatrix auprès de son époux, Arthur de Rochefide, et celui de Calyste du Guénic au foyer conjugal. Fin édifiante et cocasse à certains égards qui redistribue une fois de plus les cartes sémantiques et déclenche chez le "noble chevalier " le désillusionnement complet - après un trajet sinueux.

Calyste du Guénic, personnage "reparaissant », suit un parcours social, sentimental et initiatique qui le conduit successivement de l'hôtel du Guénic au faubourg SaintGermain en passant par le château des Touches. Force est de reconnaître qu'une transformation de son statut sémiologique et narratologique s'est opéré, inscrivant un véritable espace symbolique dans la fresque romanesque. Le jeune homme évoque l'attitude d'un lecteur, traversant les lieux successifs de l'action sans jamais s'arrêter (que l'on songe à la métaphore récurrente du «chemin » utilisée par Diderot dans Jacques le fataliste $\left.{ }^{23}\right)$; mais le lecteur poursuit seul l'activité d'interprétation et de recomposition.

Le lecteur balzacien actualise tous les savoirs précédents (historique, idéologique, intertextuel et intra-textuel). Il participe à l'évidence à l'élaboration du sens, par le biais de l'espace qui se crée. Mais les lieux qu'il préfère sont ceux qui sont bavards sur leurs conditions de représentation et qui suggèrent des pistes nouvelles : le plaisir du jeu et de l'assemblage s'en trouve accru. Le roman de Béatrix serait une «fabula ouverte", celle dans laquelle "l'intensité et la vivacité de la coopération ${ }^{24}$ sont maximales.

Par delà les savoirs accumulés et les références réactivées, la lecture semble vierge de toute hypothèse et provisoirement inédite. $\mathrm{M}$. Blanchot la conçoit ainsi, singulière et fondatrice :

Le livre qui a son origine dans l'art, n'a pas sa garantie dans le monde; et lorsqu'il est lu, il n'a encore jamais été lu, ne parvenant à sa présence d'œuvre que dans l'espace ouvert par cette lecture unique, chaque fois la première et chaque fois la seule $^{25}$.

Or, chacun sait que cette innocence-là repose sur la science - celle que donne la mémoire. "L'as-tu bien oubliée? ${ }^{26}$ Non, bien sûr. La survie du texte est à ce prix. L'espace est là - disert - qui prend le relais du discours. Et le lecteur peut, en toute quiétude, lui laisser le mot de la fin. Ce n'est pas le récit qui invente l'espace mais bien l'espace qui crée le récit.

\section{NOTES}

1. Jouve Vincent, Le lecteur, Hachette, 1993, p. 52. Nous renvoyons à l'ensemble de cet essai qui fait de précieuses mises au point sur ces questions.

2. GRACQ Julien, Préférences, Corti, 1981, p. 214.

3. de BALZAC Honoré, Avant-propos de La comédie humaine, Édition de la Pléiade, tome I, p. 7.

4. HAMON Philippe, Du descriptif, Hachette, 1993, p. 105.

5. de BALZAC H., La comédie humaine, Études de mours, Scènes de la vie privée, Béatrix, Édition de la Pléiade, tome II, p. 638. 
6. Op. cit., p. 679.

7. Op. cit., pp. 838-839.

8. Op. cit., p. 839 .

9. POULET Georges, «Lecture et interprétation du texte littéraire » in Qu'est-ce qu'un texte, Corti, 1975 , p. 67.

10. de BALZAC H., op. cit., p. 704.

11. Op. cit., p. 751.

12. B ARTHES Roland, L'obvie et l'obtus, Essais critiques III, Seuil, 1982. Le critique définit «le sens obvie » comme celui qui «s'impose, [qui] assomme » le destinataire par son évidence, (p. 46).

13. de BALZAC H., op. cit., p. 850.

14. Op. cit., p. 851.

15. Op. cit., p. 859.

16. Op. cit., p. 848.

17. En mai 1845, paraît chez Chlendowski, cette deuxième partie de Béatrix, intitulée Journal d'une lune de miel.

18. Béatrix, p. 858 .

19. Op. cit., p. 853

20. Op. cit., pp. 855-856.

21. ECO Umberto, Les limites de l'interprétation, Grasset, 1990, p. 69.

22. de BALZAC H., Avant-propos, p. 11

23. Diderot, Denis, Jacques le fataliste, La Pléiade, 1951, p. 506 : «Vous voyez, lecteur, que je suis en beau chemin, et qu'il ne tiendrait qu'à moi de vous faire attendre un an, deux ans, trois ans le récit des amours de Jacques, et en leur faisant courir à chacun tous les hasards qu'il me plairait ».

24. Eco U., Lector in fabula, Grasset, 1985, p. 159.

25. BLANCHOT Maurice, L'espace littéraire, Gallimard, 1955, p. 258.

26. de BAlZAC H., op. cit., p. 848.

\section{AUTEUR}

\section{ALINE MURA}

Institut Universitaire d'Amiens 